\title{
An evaluation of the recovery of mycobacteria from urine specimens using the automated Mycobacteria Growth Indicator Tube system (BACTEC MGIT 960) \\ Correspondence \\ Li-Hwei Sng \\ sng.li.hwei@sgh.com.sg \\ Received 4 March 2008 \\ Accepted 26 June 2008

\author{
Douglas Su Gin Chan,† May Yee Choy, Suxing Wang and Li-Hwei Sng
} \\ Central Tuberculosis Laboratory, Department of Pathology, Singapore General Hospital, Outram Road, 169608 Singapore

\begin{abstract}
The Mycobacteria Growth Indicator Tube (MGIT 960) system was evaluated against LowensteinJensen (LJ) medium and the BACTEC 460 TB system for the recovery of mycobacteria from 1393 consecutive urine specimens. The MGIT had a sensitivity of $91.3 \%$ [95 \% confidence interval (Cl), 83.2-99.4] when the combination of BACTEC 460 and $L J$ medium was used as the reference method. The mean time for positivity for MGIT and BACTEC 460 was 19.3 days and 20 days, respectively, while that for $L J$ medium was 35 days. The incidence of contamination was highest for $L$ medium ( $n=148$ ), followed by MGIT $960(n=81)$, and BACTEC 460 had the lowest incidence of contamination $(n=50)$. In conclusion, the isolation of mycobacteria from urine specimens by the MGIT 960 is comparable to that of the BACTEC 460 TB system and solid media.
\end{abstract}

\section{INTRODUCTION}

The use of automated systems in laboratories has undoubtedly decreased the turnaround time and increased the sensitivity of mycobacterial culture (NICE, 2006). We have moved ahead from traditional egg-based Lowenstein-Jensen medium culture methods to using semi-automated radiometric systems like the BD BACTEC 460, and more recently to fully automated systems like the BD BATEC Mycobacteria Growth Indicator Tube (MGIT) 960 system (hereafter referred to as MGIT 960), which function on the principle of fluorescence quenching. The MGIT 960 system had not been evaluated for mycobacterial isolation from urine specimens in the past due to lack of specimen numbers. However, a recent study by Hillemann et al. (2006) tested the MGIT 960 for recovering mycobacteria from 9558 extrapulmonary specimens, including 2069 urine samples. The incidence rate of tuberculosis in Singapore for the year 2006 was 26 cases per 100000 population (according to WHO figures). In that year, we evaluated the performance of the BD MGIT 960 in isolating mycobacteria from clinical urine specimens received in a tertiary care hospital.

\section{METHODS}

Specimens. In this study, we included all consecutive urine specimens (urine from catheter excluded) submitted to the Central

†Present address: National University Hospital, 5 Lower Kent Ridge Road, Singapore 119074.

Abbreviation: $\mathrm{Cl}$, confidence interval.
Tuberculosis Laboratory at Singapore General Hospital during the year 2006.

Culture medium inoculation, incubation and test duration. The urine was first concentrated by using a $50 \mathrm{ml}$ Falcon tube and centrifuged at $3000 \mathrm{~g}$ for $10 \mathrm{~min}$. The resulting sediment was decontaminated by the standard $\mathrm{N}$-acetyl-L-cysteine and sodium hydroxide method, with a concentration of $3 \% \mathrm{NaOH}$ (Master, 1992) (BBL MycoPrep).

After $30 \mathrm{~min}$, the suspension was centrifuged again and the sediment suspended in 1-1.5 $\mathrm{ml}$ sterile phosphate buffer ( $\mathrm{pH} \mathrm{6.8).} \mathrm{This} \mathrm{final}$ suspension was used for inoculation of the following: $0.5 \mathrm{ml}$ suspension into one MGIT 960 tube (Becton Dickinson), $0.5 \mathrm{ml}$ suspension into one BACTEC 12B vial (Becton Dickinson) (each containing polymyxin $\mathrm{B}$, amphotericin $\mathrm{B}$, nalidixic acid, trimethoprim and azlocillin - PANTA) and $0.1 \mathrm{ml}$ suspension into a Lowenstein-Jensen (LJ) slant (BBL). The remaining suspension was used for auramine smears and strand displacement amplification if requested.

The MGIT 960 tubes were incubated at $37{ }^{\circ} \mathrm{C}$ and monitored by the system for increase in fluorescence once every $60 \mathrm{~min}$ for the next 6 weeks. BACTEC 12B vials were incubated at $37 \pm 1{ }^{\circ} \mathrm{C}$ and then read by the semiautomated BACTEC 460 instrument (Becton Dickinson) twice weekly for 2 weeks and then once a week for 4 weeks thereafter. LJ slants, inoculated with $0.1 \mathrm{ml}$ processed specimen, were incubated at $37{ }^{\circ} \mathrm{C}$. The slants were examined weekly for a total of 8 weeks. All positive samples had a Kinyoun stain performed for examination of acid-fast bacilli.

Identification of mycobacteria. Mycobacterium tuberculosis complex colonies were identified using the Accuprobe (Gen-Probe), while non-tuberculous mycobacteria were identified with a variety of methods which included gene probes for Mycobacterium kansasii, Mycobacterium gordoniae and Mycobacterium avium-intracellulare 
Table 1. Isolation of mycobacteria from urine samples according to species

MTC, Mycobacterium tuberculosis complex; NTM, non-tuberculous mycobacteria.

\begin{tabular}{|c|c|c|c|c|c|c|c|}
\hline & $\begin{array}{l}\text { MGIT + ve } \\
\text { only }\end{array}$ & $\begin{array}{c}\text { BACTEC } 460 \\
+ \text { ve only }\end{array}$ & $\begin{array}{c}\text { LJ + ve } \\
\text { only }\end{array}$ & $\begin{array}{c}\text { MGIT and } \\
\text { BACTEC } 460 \\
\text { + ve only }\end{array}$ & $\begin{array}{l}\text { MGIT and } \\
\text { LJ + ve only }\end{array}$ & $\begin{array}{c}\text { BACTEC } 460 \text { and } \\
\text { LJ + ve only }\end{array}$ & All $3+$ ve \\
\hline NTM & 1 & - & 2 & 2 & 1 & - & 3 \\
\hline M. gordonae & - & - & - & 1 & - & - & - \\
\hline M. fortuitum group & 1 & - & - & - & 1 & - & - \\
\hline Total & 11 & 2 & 2 & 12 & 7 & 0 & 23 \\
\hline
\end{tabular}

complex (Gen-Probe), and high-performance liquid chromatography (Butler et al., 1996). In the event of contaminated cultures, AFBpositive specimens were reprocessed and reinoculated into BACTEC $12 \mathrm{~B}$ vials.

\section{RESULTS AND DISCUSSION}

There were 1393 urine specimens from 751 patients. Multiple specimens were received from 363 patients (2-10 per patient). We took each individual specimen to represent an independent diagnostic event. Of the 1393 urine specimens tested, $57(4.1 \%)$ specimens (from 38 patients) were positive for mycobacteria. Among these 57 specimens, 43 were found to be $M$. tuberculosis complex and 14 were non-tuberculous mycobacteria. Although the study of Hillemann et al. (2006) had more urine isolates $(n=2069)$, they had only $53(2.6 \%)$ positive mycobacterial urine cultures. This could be attributable to the lower incidence of TB in Germany, where the study took place (6.5 per 100000 population in 2006 according to WHO figures).

\section{Performance of MGIT 960 compared to BACTEC $12 B$ and $L J$ medium}

The comparison of culture methods showed that the MGIT 960 alone detected more mycobacterial isolates, with 11 isolates $(19.3 \%)$, than the BACTEC 460 alone, with 2 isolates $(3.5 \%)$, or solid LJ medium alone, with 2 isolates (3.5\%) (Table 1). In total, the MGIT 960 missed 4 isolates $(7.02 \%)$, while the combination of LJ medium and BACTEC 460 missed 11 isolates $(19.3 \%)$. This is in contrast to the study by Hillemann et al. (2006), who found that the MGIT 960 had missed $17.8 \%$ while solid medium had missed $26.7 \%$.

By comparing the proportion of mycobacteria detected by MGIT 960 vs BACTEC 460 using McNemar's test (test statistic, $\chi^{2}=11.25$, which gives $P<0.001$ at 1 degree of freedom), it is possible to reject a null hypothesis that the two systems detect the same percentage of mycobaterial isolates in urine specimens. We estimate the difference in percentage of isolates detected as $1.2 \%$ (95\% CI, 0.6$1.8 \%$ ), with the MGIT 960 detecting more isolates than the BACTEC 460. Using the BACTEC 460 alone as the reference method, the MGIT 960 had a sensitivity of 94.6\% (95\% CI, 87.3-100\%) (Table 2).

Using the combination of BACTEC 460 and LJ medium as the reference method, the MGIT had a sensitivity of $91.3 \%$ (95\% CI, 83.2-99.4\%). The MGIT was able to isolate mycobacteria in 11 cases $(0.8 \%)$ which had been negative by both the BACTEC 460 and the LJ method.

These figures are superior to a previous meta-analysis which looked at the MGIT for the isolation of mycobacteria from pulmonary and extra-pulmonary sites. In that study, the MGIT 960 had a sensitivity of $81 \%$ (95\% CI, 76-86\%) (Cruciani et al., 2004).

Instead of resorting to a fully automated system, most laboratories continue to use a combination of liquid broth and solid media for the isolation of mycobacteria. Our findings appear to support this practice for urine specimens, as the sensitivity of the MGIT was lower when a combination of LJ and BACTEC 460 was used as the reference method. However, it is plausible that unequal distribution of bacilli onto various media may influence the recovery rates and it is important to note that the

Table 2. Comparison between recovery of mycobacteria from MGIT 960 against BACTEC 460 and LJ cultures from 1393 urine samples

\begin{tabular}{|c|c|c|c|c|}
\hline & \multicolumn{2}{|c|}{$\begin{array}{c}\text { Against BACTEC } \\
460 \text { alone }\end{array}$} & \multicolumn{2}{|c|}{$\begin{array}{c}\text { Against BACTEC } \\
460 \text { and LJ }\end{array}$} \\
\hline & $\begin{array}{l}\text { BACTEC } \\
460+\mathrm{ve}\end{array}$ & $\begin{array}{c}\text { BACTEC } \\
460-\text { ve }\end{array}$ & $\begin{array}{c}\text { BACTEC } \\
460 \text { and } \\
\text { LJ + ve }\end{array}$ & $\begin{array}{c}\text { BACTEC } \\
460 \text { and } \\
\text { LJ -ve }\end{array}$ \\
\hline MGIT + ve & 35 & 18 & 42 & 11 \\
\hline MGIT -ve & 2 & 1338 & 4 & 1336 \\
\hline Total & 37 & 1356 & 46 & 1347 \\
\hline
\end{tabular}


Table 3. Time to positivity

\begin{tabular}{|lccccc|}
\hline & Mean (days) & Mode (days) & Median (days) & Min. time (days) & Max. time (days) \\
\hline MGIT 960 & 19.3 & 15 & 15 & 0 & 42 \\
BACTEC 460 & 20.0 & 14 & 20 & 4 & 41 \\
LJ slant & 37.5 & 35 & 35 & 21 & 53 \\
\hline
\end{tabular}

significance of the non-tuberculous mycobacteria isolated by the solid medium alone was not evident upon clinical review during the period of the study.

\section{Time to positivity}

The appeal of both the BACTEC 460 and the MGIT 960 is the decreased turnaround time for mycobacterial isolation. This advantage would enable clinicians to establish a definitive diagnosis, and institute appropriate therapy and infection control measures within the shortest possible time. We found that the times for positivity for MGIT 960 and BACTEC 460 were comparable, with a mean time of 19.3 days and 20.0 days, respectively $(P=0.6)$ (Table 3$)$. Not surprisingly, solid medium had a significantly longer time to positivity (mean of 37.5 days). In a previous study by Somoskovi et al. (2000), which looked at pulmonary specimens, the MGIT had the shortest time to positivity, followed by the BACTEC 12B and LJ medium (mean of 13.2 days vs 16.8 days and 36.2 days, respectively). A possible reason for the longer time to positivity for urine in the MGIT in our study could be the presence of inhibitory substances in urine (i.e. antimicrobials which may have been commenced before the diagnosis of renal tuberculosis). The smaller volume of specimen added could have accounted for the reduced sensitivity and increased turnaround time for the LJ method.

\section{Contamination}

Decontamination of specimens was performed using $3 \%$ $\mathrm{NaOH}$ in our centre as this protocol resulted in an overall contamination rate of $2-5 \%$, which was consistent with previous data (Collins et al., 1997) for all specimens in our laboratory. We found that the rate of breakthrough contamination (presumptive positives which were actually found to be due to non-mycobacterial organisms) were $10.6 \%(n=148)$ for LJ medium (95\% CI, 9-12.24\%), $5.8 \%(n=81)$ for the MGIT (95\% CI, $4.58-7.04 \%)$ and $3.6 \%(n=50)$ for the BACTEC 460 (95\% CI, $2.61-4.57 \%$ ).

This is in contrast to the study of Hillemann et al. (2006), which found the rate of contamination in the MGIT to be greater than that of LJ medium for urine specimens ( $14.9 \%$ vs $7.2 \%$ ). In that study, $1 \% \mathrm{NaOH}$ was used for decontamination, which probably accounted for the higher rate of contamination in the MGIT. However, our figures were comparable with a previous meta-analysis by Cruciani et al.
(2004), which showed a contamination rate of $12.8 \%$ with solid medium, $8.6 \%$ for MGIT and $4.4 \%$ for BACTEC 460 .

Our findings remain limited by the fact that although a large number of urine specimens were included in the study, the actual number of specimens in which mycobacteria was detected remained small. Perhaps future studies should include urine specimens collected over a longer period. In summary, our study has shown that isolation of mycobacteria from urine by the MGIT 960 system is comparable if not superior to that of the BACTEC 460 system and solid medium.

\section{ACKNOWLEDGEMENTS}

We thank the staff of the Central TB Laboratory for their technical assistance; Mr Kai Ming Tay, and Ms Catherine Mary Zi Hui Chua, for helping in the retrieval of data in this study.

\section{REFERENCES}

Butler, W. R., Floyd, M. M., Silcox, V., Cage, G., Desmond, E. \& other authors (1996). Standardized Method for HPLC Identification of Mycobacteria. Atlanta, GA: Centers for Disease Control and Prevention.

Collins, C. H., Grange, J. M. \& Yates, M. D. (1997). Tuberculosis Bacteriology: Organisation and Practice, 2nd edn. Butterworth Heinemann London.

Cruciani, M., Scarparo, C., Malena, M., Bosco, O., Serpelloni, G. \& Mengoli, C. (2004). Meta-analysis of BACTEC MGIT 960 and BACTEC $460 \mathrm{~TB}$, with or without solid media for detection of mycobacteria. J Clin Microbiol 42, 2321-2325.

Hillemann, D., Richter, E. \& Rüsch-Gerdes, S. (2006). Use of the BACTEC Mycobacteria Growth Indicator Tube 960 automated system for recovery of mycobacteria from 9,558 extrapulmonary specimens, including urine samples. J Clin Microbiol 44, 4014-4017.

Master, R. N. (1992). Mycobacteriology. In Clinical Microbiology Procedures Handbook. Edited by H. D. Isenberg. Washington, DC: ASM Press.

NICE (2006). Tuberculosis: Clinical Diagnosis and Management of Tuberculosis, and Measures for its Prevention and Control. London, UK: National Institute for Health and Clinical Excellence.

Somoskovi, A., Kodmon, C., Lantos, A., Bartfai, Z., Tamasi, L., Fuzy, J. \& Magyar, P. (2000). Comparison of recoveries of Mycobacterium tuberculosis using the automated BACTEC MGIT 960 system, the BACTEC 460 TB system, and Lowenstein-Jensen medium. J Clin Microbiol 38, 2395-2397. 